\title{
FLUOROUS SUPPORTED SYNTHESIS OF PYRAZOLONE DERIVATIVES FROM ALLYLIC ALCOHOLS USING A PALLADIUM-CATALYZED STRATEGY
}

Nicolas Gouault ${ }^{\mathrm{a}, *}$, Alexandre Cariou ${ }^{\mathrm{a}}$, Jean-François Cupifa, Patrícia de Aguiar Amaral ${ }^{\mathrm{b}}$ and Michèle David ${ }^{\mathrm{a}}$

aEquipe PNSCM, UMR 6226, ISCR, Université de Rennes1, 35000 Rennes, France

${ }^{b}$ LaPlaM/PPGCA, Universidade do Extremo Sul Catarinense, 88806-000 Criciúma - SC, Brasil

Recebido em 23/12/2015; aceito em 28/03/2016; publicado na web em 06/06/2016

\begin{abstract}
Ten substituted pyrazolone derivatives were easily synthesized from perfluorinated allylic alcohols, via a straightforward two steps reaction involving a Heck-coupling/isomerization reaction and subsequent condensation of hydrazines. This fluorous supported methodology, that combines a cyclo-release approach and F-SPE purification allows for the obtaining of the final products with a good purity.
\end{abstract}

Keywords: pyrazolones; fluorous tag; Heck coupling; allylic alcohols.

\section{INTRODUCTION}

Pyrazolones represent an important class of molecules that have been well-studied because of their relevant biological activities. ${ }^{1-4}$ They were first extensively developed for their anti-inflammatory, ${ }^{5}$ analgesic ${ }^{6}$ and antipyretic ${ }^{7}$ properties. More recently, great attention has been paid to their versatile role as antimicrobial, ${ }^{8}$ antiparasitic, ${ }^{9}$ antiviral ${ }^{10}$ and antineoplastic ${ }^{11}$ agents. For instance, edaravone (1) is a potent drug used in the treatment of brain ischemia ${ }^{12}$ and was shown to alleviate Alzheimer's disease-type pathologies, ${ }^{13}$ metamidole (2) is known to have antipyretic and analgesic activities, ${ }^{7}$ the pyrazole (3) is known to possess HIV-1 integrase inhibitory properties ${ }^{14}$ and (4) has idiopathic pulmonary fibrosis activity ${ }^{15}$ (Figure 1).

The most common strategy (scheme 1) to obtain pyrazolones is the Knorr condensation of substituted hydrazines with $\beta$-ketoesters, ${ }^{16}$ the latter generally being obtained by Claisen condensation ${ }^{17}$ or from Meldrum's acid. ${ }^{18,19} \mathrm{~A}$ more recent approach to such $\beta$-ketoesters intermediates is the conversion of allylic alcohols to ketone in a Heck coupling process. ${ }^{20,21}$ It is obvious that described methods produce unwanted by-products (competition between $2 \beta$-hydride eliminations in this process). In this respect, the development of an approach that allows an easy elimination of these would be of significance.

Nowadays, in the field of combinatorial chemistry, one most attractive and versatile tool for the solid-phase parallel synthesis of pharmacologically active heterocyclic compounds is the cyclo-elimination strategy. ${ }^{22}$ Such an approach involves the simultaneous cyclization and release of the heterocyclic compound from the solid support resulting in the obtaining of the final product with the adequate purity for a biological evaluation, intermediates or by-products remaining on the support.

We have previously reported the synthesis of diverse heterocycles using such cyclo-release approaches and fluorous tags, ${ }^{23-25}$ a methodological tool that was mainly developed to simplify the purification procedure in the parallel synthesis strategies. ${ }^{26-29}$ We herein report an efficient synthetic route to some structurally diverse pyrazolone derivatives from perfluorinated allylic alcohols. We investigated the palladium-catalyzed transformation of allylic alcohols to $\beta$-ketoesters and their subsequent cyclization into pyrazolones. Besides the practical benefits of fluorous-phase reactions, heterocyclization-cleavage strategy afford the opportunity to provide a final product with high purity. Our synthetic approach is outlined in scheme 1.

Baylis-Hillman adducts and allylic alcohols are of particular interest as starting building blocks in the Heck coupling reaction since the $\beta$-hydride elimination of the organopalladium intermediate gives rise, in a regioselective manner, to $\beta$-keto ester derivatives, which upon reaction with hydrazines may give pyrazolones in a cyclative cleavage. Each step allows the introduction of diversity and purification steps can be considerably simplified. We expect this method to find extensive application in the fields of combinatorial chemistry, diversity-oriented synthesis and drug discovery.<smiles>CC1=NN(c2ccccc2)C(=O)C1</smiles>

Edaravone (1)
(Brain ischemia)<smiles>Cc1c(C(C)CS(=O)(=O)O[Na])c(=O)n(-c2ccccc2)n1C</smiles>

Metamizole (2) (analgesic)<smiles>O=C(O)C1=NN(c2cccc([N+](=O)[O-])c2)C(=O)/C1=C\c1ccc(Oc2ccc(F)cc2)c(O)c1</smiles>

(3)

(HIV-1 Integrase inhibitor)<smiles>[R2]c1c2c(=O)n([R1])n([R4])c2cc(=O)n1[R]</smiles>

(4)

(Idiopathic pulmonary fibrosis)

Figure 1. Representative examples of biologically active pyrazolone derivatives

*e-mail: nicolas.gouault@univ-rennes1.fr 


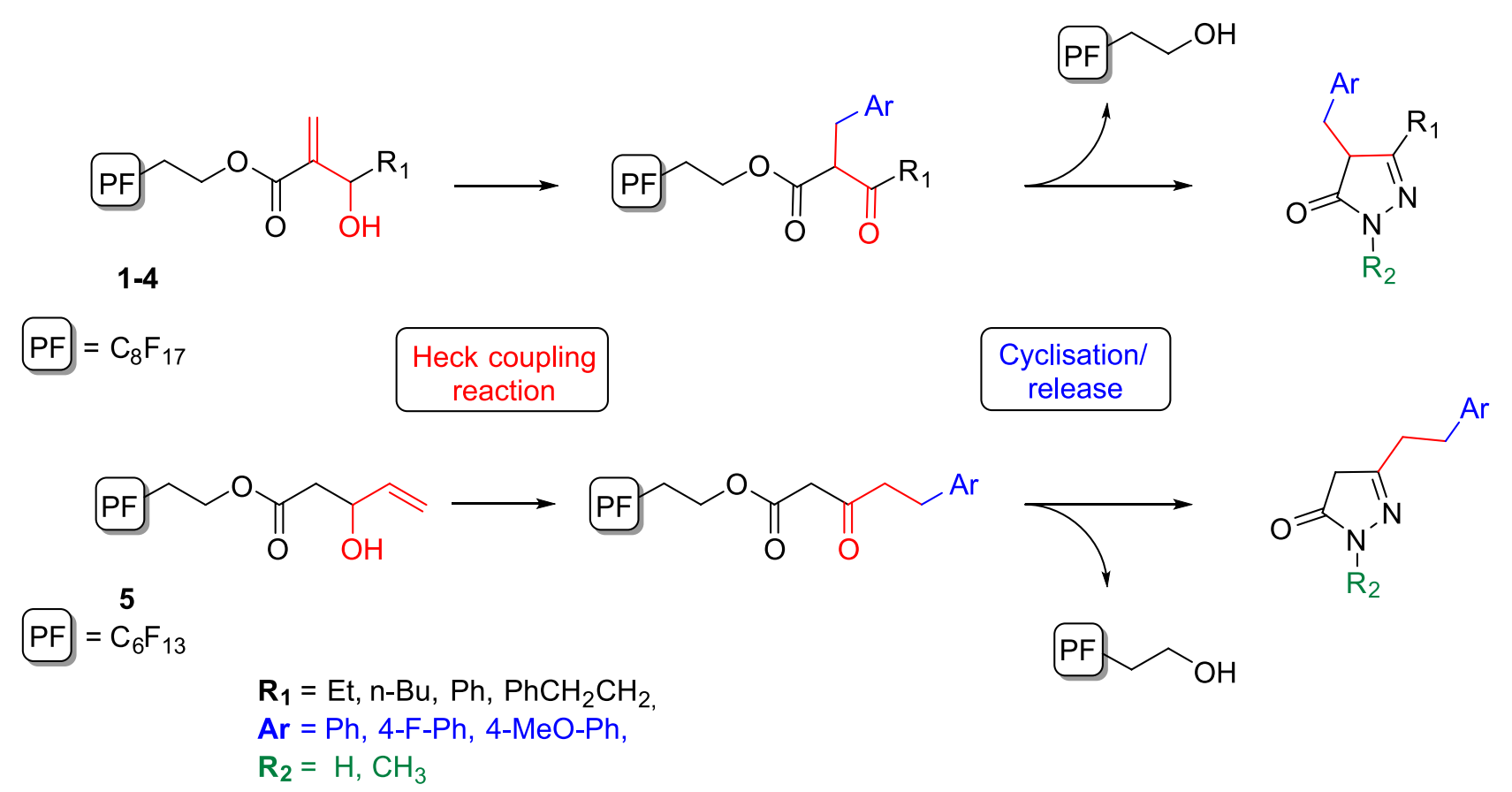

Scheme 1. Synthetic approach to pyrazolone derivatives from fluorous-supported allylic alcohols

\section{EXPERIMENTAL}

\section{General}

All reagents of high quality were purchased from commercial suppliers, and used without further purification. All reactions requiring anhydrous conditions were performed under an argon atmosphere using oven dried glassware. DMF and THF were distilled from $\mathrm{CaH}_{2}$ and $\mathrm{Na}$ /benzophenone, respectively. ${ }^{1} \mathrm{H}$ and ${ }^{13} \mathrm{C}$ NMR were recorded at 300 and $75 \mathrm{MHz}$, respectively, on a Bruker AM 300 spectrometer or at $270 \mathrm{MHz}$ on a Jeol GSX $270 \mathrm{WB}$, using $\mathrm{CDCl}_{3}$ (and TMS as internal standard) or DMSO- $d_{6} . \delta$ values are given in parts per million (ppm), coupling constants ( $J$ values) are given in Hertz $(\mathrm{Hz})$, and multiplicity of signals are reported as follows: s, singlet; d, doublet; t, triplet; q, quadruplet; m, multiplet; bs, broad singlet. Thin layer chromatography was performed using precoated silica gel plate $(0.2$ $\mathrm{mm}$ thickness). All melting points are uncorrected. IR spectra were run on a PerkinElmer Spectrum 16 PC spectrometer; only selected absorbances are quoted. HRMS (EI) measurements were recorded on a Varian MAT 311 mass spectrometer at the Centre Régional de Mesures Physique de l'Ouest. F-SPE was performed using silica gel 60 C8-reversed phase perfluorinated $(35-70 \mu \mathrm{m}$, Fluka), which was conditioned in a cartridge for under vacuum filtration.

\section{Chemistry}

\section{Synthesis of Baylis-Hillman adducts (1-4)}

To a 3,3,4,4,5,5,6,6,7,7,8,8,9,9,10,10,10-heptadecafluorodecyl-acrylate (2 g, $3.86 \mathrm{mmol}, 1 \mathrm{eq}$ ) was added DABCO (490 mg, 3.86 $\mathrm{mmol}, 1 \mathrm{eq})$ and 2 eq of aldehyde $\left(\mathrm{R}_{1} \mathrm{CHO}\right)$ in neat conditions. After stirring at room temperature for $48 \mathrm{~h}$, the crude reaction mixture is diluted in $\mathrm{CH}_{2} \mathrm{Cl}_{2}(30 \mathrm{~mL})$, washed three times with water and dried over anhydrous sodium sulphate. After filtration, the mixture was evaporated in vacuo, and then dissolved in $10 \mathrm{~mL}$ of [80/20] $\mathrm{MeOH} /$ $\mathrm{H}_{2} \mathrm{O}(\mathrm{v} / \mathrm{v}) .2 \mathrm{~mL}$ of this material was applied to the top of a fluorous silica gel cartridge $(3 \mathrm{~g})$ which had been preconditioned with [80/20] $\mathrm{MeOH} / \mathrm{H}_{2} \mathrm{O}$. The cartridge was eluted with [80/20] $\mathrm{MeOH} / \mathrm{H}_{2} \mathrm{O}(2 \mathrm{x}$
$10 \mathrm{~mL})$, then with $\mathrm{CH}_{2} \mathrm{Cl}_{2}(2 \times 10 \mathrm{~mL})$. This operation was repeated 5 times on the same cartridge. The $\mathrm{CH}_{2} \mathrm{Cl}_{2}$ fractions were then combined and removal of the solvent in vacuo afforded a mixture of the desired fluorous Baylis-Hillman adduct and the fluorous alcohol.

Perfluorinated Baylis-Hillman adduct (1): pale yellow oil (82\%). ${ }^{1} \mathbf{H}$ NMR $\left(270 \mathrm{MHz}, \mathrm{CDCl}_{3}\right): \delta_{\mathrm{H}} 0.96\left(3 \mathrm{H}, \mathrm{t}, \mathrm{H}_{5}, J_{\mathrm{H} 5-\mathrm{H} 4}=7.4 \mathrm{~Hz}\right), 1.60-$ $1.78\left(2 \mathrm{H}, \mathrm{m}, \mathrm{H}_{4}\right), 2.53\left(2 \mathrm{H}, \mathrm{tt}, \mathrm{H}_{\mathrm{b}}, J_{\mathrm{Hb}-\mathrm{Ha}}=6.4 \mathrm{~Hz}, J_{\mathrm{Hb}-\mathrm{F}}=18.1 \mathrm{~Hz}\right)$, $2.61(1 \mathrm{H}, \mathrm{bs},-\mathrm{OH}), 4.33-4.39\left(1 \mathrm{H}, \mathrm{m}, \mathrm{H}_{3}\right), 4.49\left(2 \mathrm{H}, \mathrm{t}, \mathrm{H}_{\mathrm{a}}, J_{\mathrm{Ha}-\mathrm{Hb}}=6.4\right.$ $\mathrm{Hz}), 5.87\left(1 \mathrm{H}, \mathrm{s},=\mathrm{CH}_{2}\right), 6.28\left(1 \mathrm{H}, \mathrm{s},=\mathrm{C}_{2}\right) .{ }^{13} \mathrm{C} \mathrm{NMR}(67.5 \mathrm{MHz}$, $\left.\mathrm{CDCl}_{3}\right): \delta_{\mathrm{C}} 10.1\left(\mathrm{C}_{5}\right), 29.2\left(\mathrm{C}_{4}\right), 30.6\left(\mathrm{C}_{\mathrm{b}}, \mathrm{t}, J_{\mathrm{Cb}-\mathrm{F}}=21.8 \mathrm{~Hz}\right), 56.7\left(\mathrm{C}_{\mathrm{a}}\right.$, bs), 72.9( $\left.\mathrm{C}_{3}\right), 126.0\left(=\mathrm{CH}_{2}\right), 142.0\left(\mathrm{C}_{2}\right), 166.1\left(\mathrm{C}_{1}\right) .{ }^{19} \mathbf{F}$ NMR $(470.6$ $\left.\mathrm{MHz}, \mathrm{CDCl}_{3}\right): \delta_{\mathrm{F}}-80.8(3 \mathrm{~F}),-113.6(2 \mathrm{~F}),-121.7(2 \mathrm{~F}),-121.9(4 \mathrm{~F})$, -122.8 (2F), -123.6 (2F), -126.2 (2F). FTIR: 1100 to 1300 (CF), 1629 $(\mathrm{C}=\mathrm{C}$ methylene $), 1719(\mathrm{CO}), 3430(\mathrm{OH})$. HRMS $(\mathbf{E I}):(\mathrm{m} / \mathrm{z}) \mathrm{calcd}$ for $\mathrm{C}_{16} \mathrm{H}_{13} \mathrm{~F}_{17} \mathrm{O}_{3}$ 576.0593, found 576.0504.

Perfluorinated Baylis-Hillman adduct (2): pale yellow oil (72\%). ${ }^{1} \mathbf{H}$ NMR $\left(270 \mathrm{MHz}, \mathrm{CDCl}_{3}\right): \delta_{\mathrm{H}} 0.90\left(3 \mathrm{H}, \mathrm{t}, \mathrm{H}_{7}, J_{\mathrm{H} 7 \mathrm{H} 6}=6.8 \mathrm{~Hz}\right)$, $1.26-1.73\left(6 \mathrm{H}, \mathrm{m}, \mathrm{H}_{4,5,6}\right), 2.42(1 \mathrm{H}, \mathrm{bs},-\mathrm{OH}), 2.53\left(2 \mathrm{H}, \mathrm{tt}, \mathrm{H}_{\mathrm{b}}, J_{\mathrm{Hb}-\mathrm{Ha}}\right.$ $\left.=6.4 \mathrm{~Hz}, J_{\mathrm{Hb}-\mathrm{F}}=18.1 \mathrm{~Hz}\right), 4.38-4.45\left(1 \mathrm{H}, \mathrm{m}, \mathrm{H}_{3}\right), 4.49\left(2 \mathrm{H}, \mathrm{t}, \mathrm{H}_{\mathrm{a}}\right.$, $\left.J_{\mathrm{Ha}-\mathrm{Hb}}=6.4 \mathrm{~Hz}\right), 5.87\left(1 \mathrm{H}, \mathrm{s},=\mathrm{C}_{2}\right), 6.26\left(1 \mathrm{H}, \mathrm{s},=\underline{\mathrm{C}}_{2}\right) .{ }^{13} \mathbf{C ~ N M R}$ $\left(67.5 \mathrm{MHz}, \mathrm{CDCl}_{3}\right): \delta_{\mathrm{C}} 14.0\left(\mathrm{C}_{7}\right), 22.5\left(\mathrm{C}_{6}\right), 28.0\left(\mathrm{C}_{5}\right), 30.6\left(\mathrm{C}_{\mathrm{b}}, \mathrm{t}\right.$, $\left.J_{\mathrm{Cb}-\mathrm{F}}=21.8 \mathrm{~Hz}\right), 36.0\left(\mathrm{C}_{4}\right), 56.7\left(\mathrm{C}_{\mathrm{a}}, \mathrm{bs}\right), 71.6\left(\mathrm{C}_{3}\right), 125.8\left(=\mathrm{CH}_{2}\right)$, $142.3\left(\mathrm{C}_{2}\right), 166.1\left(\mathrm{C}_{1}\right),{ }^{19} \mathbf{F}$ NMR $\left(470.6 \mathrm{MHz}, \mathrm{CDCl}_{3}\right): \delta_{\mathrm{F}}-80.9$ (3F), -113.6 (2F), -121.7 (2F), -122.0 (4F), -122.8 (2F), -123.6 (2F), -126.2 (2F). FTIR: 1100 to $1300(\mathrm{CF}), 1627$ (C=C methylene), $1720(\mathrm{CO}), 3425(\mathrm{OH})$. HRMS: $(\mathrm{m} / \mathrm{z})$ calcd for $\mathrm{C}_{18} \mathrm{H}_{17} \mathrm{~F}_{17} \mathrm{O}_{3}$ 604.0906, found 604.0862.

Perfluorinated Baylis-Hillman adduct (3): white solid (85\%). ${ }^{1} \mathbf{H}$ NMR $\left(270 \mathrm{MHz}, \mathrm{CDCl}_{3}\right): \delta_{\mathrm{H}} 2.43\left(2 \mathrm{H}, \mathrm{tt}, \mathrm{Hb}, J_{\mathrm{Hb}-\mathrm{Ha}}=6,4 \mathrm{~Hz}\right.$, $\left.J_{\mathrm{Hb}-\mathrm{F}}=18.1 \mathrm{~Hz}\right), 2.80(1 \mathrm{H}, \mathrm{bs},-\mathrm{OH}), 4.42\left(2 \mathrm{H}, \mathrm{t}, \mathrm{H}_{\mathrm{a}}, J_{\mathrm{Ha}-\mathrm{Hb}}=6.4 \mathrm{~Hz}\right)$, $5.58\left(1 \mathrm{H}, \mathrm{s}, \mathrm{H}_{3}\right), 5.93\left(1 \mathrm{H}, \mathrm{s},=\mathrm{CH}_{2}\right), 6.38\left(1 \mathrm{H}, \mathrm{s},=\mathrm{C}_{2}\right), 7.30-7.37$ $\left(5 \mathrm{H}, \mathrm{m}, \mathrm{H}_{\text {arom }}\right) \cdot{ }^{13} \mathrm{C}$ NMR $\left(67.5 \mathrm{MHz}, \mathrm{CDCl}_{3}\right): \delta_{\mathrm{C}} 30.4\left(\mathrm{C}_{\mathrm{b}}, \mathrm{t}, J_{\mathrm{Cb}-\mathrm{F}}\right.$ $=21.8 \mathrm{~Hz}), 56.7\left(\mathrm{C}_{\mathrm{a}}, \mathrm{bs}\right), 73.1\left(\mathrm{C}_{3}\right), 126.6 ; 126.9 ; 128.0 ; 128.5$ $\left(=\underline{\mathrm{CH}}_{2}\right.$ and $\left.\mathrm{CH}_{\text {arom }}\right), 141.0\left(\mathrm{C}_{\mathrm{IV}}\right), 141.5\left(\mathrm{C}_{\mathrm{IV}}\right), 165.7\left(\mathrm{C}_{1}\right) .{ }^{19} \mathbf{F} \mathbf{N M R}$ $\left(470.6 \mathrm{MHz}, \mathrm{CDCl}_{3}\right): \delta_{\mathrm{F}}-80.8(3 \mathrm{~F}),-113.6(2 \mathrm{~F}),-121.6(2 \mathrm{~F})$, 
-121.9 (4F), -122.7 (2F), -123.5 (2F), -126.1 (2F). FTIR: 1100 to $1300(\mathrm{CF}), 1602(\mathrm{C}=\mathrm{C}$ arom $), 1630(\mathrm{C}=\mathrm{C}$ methylene $), 1718(\mathrm{CO})$, $3470(\mathrm{OH})$. HRMS (EI): $(\mathrm{m} / \mathrm{z})$ calcd for $\mathrm{C}_{20} \mathrm{H}_{13} \mathrm{~F}_{17} \mathrm{O}_{3} 624.0593$, found 624.0642 .

Perfluorinated Baylis-Hillman adduct (4): colorless oil (72\%). ${ }^{1} \mathbf{H}$ NMR $\left(270 \mathrm{MHz}, \mathrm{CDCl}_{3}\right): \delta_{\mathrm{H}} 1.70(1 \mathrm{H}, \mathrm{bs},-\mathrm{OH}), 1.88-2.10(2 \mathrm{H}$, $\left.\mathrm{m}, \mathrm{H}_{4}\right), 2.38-3.00\left(4 \mathrm{H}, \mathrm{m}, \mathrm{H}_{5, \mathrm{~b}}\right), 4.42-4.47\left(3 \mathrm{H}, \mathrm{m}, \mathrm{H}_{3, \mathrm{a}}\right), 5.87(1 \mathrm{H}$, $\left.\mathrm{s},=\mathrm{CH}_{2}\right), 6.27\left(1 \mathrm{H}, \mathrm{s},=\mathrm{C}_{2}\right), 7.10-7.30\left(5 \mathrm{H}, \mathrm{m}, \mathrm{H}_{\text {arom }}\right) .{ }^{13} \mathbf{C} \mathbf{~ N M R}$ $\left(67.5 \mathrm{MHz}, \mathrm{CDCl}_{3}\right): \delta_{\mathrm{C}} 30.5\left(\mathrm{C}_{\mathrm{b}}, \mathrm{t}, J_{\mathrm{Cb}-\mathrm{F}}=21.8 \mathrm{~Hz}\right), 32.1\left(\mathrm{C}_{4}\right), 37.8$ $\left(\mathrm{C}_{5}\right), 56.8\left(\mathrm{C}_{\mathrm{a}}, \mathrm{bs}\right), 70.8\left(\mathrm{C}_{3}\right), 126.0 ; 126.1 ; 128.5 ; 128.6\left(=\underline{\mathrm{CH}}_{2}\right.$ and $\left.\mathrm{CH}_{\text {arom }}\right), 141.6\left(\mathrm{C}_{\mathrm{IV}}\right), 142.1\left(\mathrm{C}_{\mathrm{IV}}\right), 166.0\left(\mathrm{C}_{1}\right) .{ }^{19} \mathrm{~F} \mathrm{NMR}(470.6 \mathrm{MHz}$, $\left.\mathrm{CDCl}_{3}\right): \delta_{\mathrm{F}}-80.8(3 \mathrm{~F}),-113.6(2 \mathrm{~F}),-121.7(2 \mathrm{~F}),-121.9(4 \mathrm{~F}),-122.7$ (2F), $-123.5(2 \mathrm{~F}),-126.2(2 \mathrm{~F})$. FTIR: 1100 to $1300(\mathrm{CF}), 1603(\mathrm{C}=\mathrm{C}$ arom), 1628 (C=C methylene), $1718(\mathrm{CO}), 3420(\mathrm{OH})$. HRMS (EI): $(\mathrm{m} / \mathrm{z})$ calcd for $\left[\mathrm{M}-\mathrm{H}_{2} \mathrm{O}\right]^{+} \mathrm{C}_{22} \mathrm{H}_{15} \mathrm{~F}_{17} \mathrm{O}_{2634.0801}$, found 634.0829.

\section{Synthesis of perfluorinated allylic alcohol 5}

To a stirred solution of $5 \mathrm{~g}$ of $1 \mathrm{H}, 1 \mathrm{H}, 2 \mathrm{H}, 2 \mathrm{H}$-perfluorooctanol (13.7 mmol) in $90 \mathrm{~mL}$ of dry $\mathrm{CH}_{2} \mathrm{Cl}_{2}$, under inert atmosphere, were added freshly distilled triethylamine (1.2 eq., $2.3 \mathrm{~mL}$ ), then slowly acetyl chloride (1.2 eq., $1.2 \mathrm{~mL}$ ) in $10 \mathrm{~mL}$ of dry $\mathrm{CH}_{2} \mathrm{Cl}_{2}$. After $18 \mathrm{~h}$ at room temperature, the reaction mixture was washed four times with $30 \mathrm{~mL}$ of water and this aqueous layer was extracted two times with $30 \mathrm{~mL}$ of $\mathrm{CH}_{2} \mathrm{Cl}_{2}$. The combined organic layers were dried $\left(\mathrm{Na}_{2} \mathrm{SO}_{4}\right)$ and evaporated under reduced pressure to give the polyfluorooctyl acetate $(98 \%)$.

1H,1H,2H,2H-perfluorooctyl acetate: colorless oil; FTIR: 1145 to 1234 (CF), 1369 (-OC-), 1751 (-OCO-) $\mathrm{cm}^{-1},{ }^{1} \mathbf{H}$ NMR (270 $\left.\mathrm{MHz}, \mathrm{CDCl}_{3}\right): \delta_{\mathrm{H}} 2.00\left(3 \mathrm{H}, \mathrm{s}, \mathrm{H}_{2}\right) ; 2.45\left(2 \mathrm{H}, \mathrm{m}, \mathrm{H}_{2}\right) ; 4.38(2 \mathrm{H}, \mathrm{t}$, $\left.\mathrm{H}_{1}, J=6.75 \mathrm{~Hz}\right) ;{ }^{13} \mathbf{C}$ NMR $\left(67.5 \mathrm{MHz}, \mathrm{CDCl}_{3}\right): \delta_{\mathrm{C}} 20.7\left(\mathrm{C}_{2}\right), 30.5$ $\left(\mathrm{C}_{2}\right), 56.3\left(\mathrm{C}_{1}\right), 170.6\left(\mathrm{C}_{1}\right) ;{ }^{19} \mathbf{F}$ NMR $\left(254 \mathrm{MHz}, \mathrm{CDCl}_{3}\right): \delta_{\mathrm{F}}-81.3$ (3F), -114.2 (2F), -122.4 (2F), -123.4 (2F), -124.1 (2F), -126.7 (2F). HRMS (EI): $(\mathrm{m} / \mathrm{z})$ calcd for $\mathrm{C}_{19} \mathrm{H}_{32} \mathrm{O}_{4} 324.2301$, found 324.2291 . TLC: DCM $(100 \%) \mathrm{R}_{f}=0.80$.

To a stirred solution of polyfluorooctyl acetate $(2 \mathrm{~g}, 5.0 \mathrm{mmol})$ in dry THF $(50 \mathrm{~mL})$, under inert atmosphere, was added a solution of LHMDS (5.2 mL, $1.1 \mathrm{eq})$ at $-78^{\circ} \mathrm{C}$ and the resulting solution was stirred for 45 minutes. Then acrolein $(0.5 \mathrm{~mL}, 1.5$ eq. $)$ and, ten minutes later, $\mathrm{TMSCl}(0.6 \mathrm{~mL}, 1.0$ eq. $)$ were added and the resulting solution was stirred for 4 hours at $-78^{\circ} \mathrm{C}$. The reaction was then quenched by addition of sat. $\mathrm{NH}_{4} \mathrm{Cl}$ followed by addition of $1 \mathrm{~mol} \mathrm{~L}^{-1} \mathrm{HCl}$ until the $\mathrm{pH}$ was acid. The mixture was extracted with diethyl ether. The organic layers were dried and evaporated under reduced pressure. The residue was purified through fluorous flash chromatography to give the ester $(70 \%)$.

1H,1H,2H,2H-perfluorooctyl-3-hydroxypent-4-enoate (5): yellow oil; FTIR: 1144 to $1236(\mathrm{CF}), 1740$ (-OCO-), $3435(-\mathrm{OH})$ $\mathrm{cm}^{-1}$.; ${ }^{1} \mathbf{H}$ NMR $\left(270 \mathrm{MHz}, \mathrm{CDCl}_{3}\right) \delta_{\mathrm{H}} 2.20-2.60\left(4 \mathrm{H}, \mathrm{m}, \mathrm{H}_{2}\right.$ and $\mathrm{H}_{2}$ ), $4.28\left(2 \mathrm{H}, \mathrm{t}, \mathrm{H}_{1}, J_{\mathrm{H}^{\prime}-\mathrm{H}{ }^{\prime}}=6.54 \mathrm{~Hz}\right), 4.50\left(1 \mathrm{H}, \mathrm{m}, \mathrm{H}_{3}\right), 5.30(1 \mathrm{H}, \mathrm{dt}$, $\left.\mathrm{H}_{5}, J_{\mathrm{H} 5-\mathrm{H} 4}=17.2 \mathrm{~Hz}, 1.2 \mathrm{~Hz}\right), 5.70-5.95\left(1 \mathrm{H}, \mathrm{ddd}, \mathrm{H}_{4}, J_{\mathrm{H} 4-\mathrm{H} 5}=17.1\right.$ $\mathrm{Hz}, 10.6 \mathrm{~Hz}, 5.7 \mathrm{~Hz}) ;{ }^{13} \mathrm{C} \mathrm{NMR}\left(67.8 \mathrm{MHz}, \mathrm{CDCl}_{3}\right): \delta_{\mathrm{C}} 30.5\left(\mathrm{C}_{2}\right)$, $41.1\left(\mathrm{C}_{2}\right), 56.6\left(\mathrm{C}_{1}\right), 68.9\left(\mathrm{C}_{3}\right), 115.7\left(\mathrm{C}_{5}\right), 138.6\left(\mathrm{C}_{4}\right), 171.6\left(\mathrm{C}_{1}\right)$, ${ }^{19} \mathbf{F}$ NMR $\left(254 \mathrm{MHz}, \mathrm{CDCl}_{3}\right): \delta_{\mathrm{F}}-81.3(3 \mathrm{~F}),-114.2(2 \mathrm{~F}),-122.4$ (2F), -123.4 (2F), -124.1 (2F), -126.7 (2F). TLC: DCM/AcOEt $(90 / 10) \mathrm{R}_{f}=0.40$.

\section{Representative procedure for the Heck coupling/isomerization reaction from $B H$ adducts}

To a stirred solution of Baylis-Hillman adduct $\mathbf{1}$ (270 mg, 0.47 $\mathrm{mmol}$ ) in $\mathrm{THF}$ ( $3 \mathrm{~mL}$ ) was added $\mathrm{NaHCO}_{3}(138 \mathrm{mg}, 3.5$ eq. $), \mathrm{NBu}_{4} \mathrm{Br}$
(151 mg, 1 eq) and iodoanisole (329 mg, $3.0 \mathrm{eq})$. Then, the solution was degassed with nitrogen and the catalyst $\left[\mathrm{Pd}(\mathrm{OAc})_{2}(5 \mathrm{mg}, 5\right.$ mol\%)] was added. After the reaction mixture was stirred 18 hours under reflux in an oil bath, the crude mixture was allowed to cool at room temperature, and then diluted in $1 \mathrm{~mL}$ of diethyl ether. This suspension was applied over diatomaceous earth and washed with diethyl ether $(3 \times 10 \mathrm{~mL})$. The filtrate was then washed three times with water and dried over anhydrous sodium sulphate. After filtration, the mixture was evaporated in vacuo, and then dissolved in $1 \mathrm{~mL}$ of $[80 / 20] \mathrm{MeOH} / \mathrm{H}_{2} \mathrm{O}$. The material was applied to the top of a fluorous silica gel cartridge ( $3 \mathrm{~g})$ which had been preconditioned with [80/20] $\mathrm{MeOH} / \mathrm{H}_{2} \mathrm{O}$. The cartridge was eluted with [80/20] $\mathrm{MeOH} / \mathrm{H}_{2} \mathrm{O}(2 \mathrm{x}$ $10 \mathrm{~mL})$, then diethyl ether $(2 \times 10 \mathrm{~mL})$. The combined diethyl ether fractions were dried over anhydrous sodium sulfate. After filtration, solvent evaporation resulted in the desired perflurinated $\beta$-keto ester $6(90 \%)$.

$1 H, 1 H, 2 H, 2 H$-perfluorodecyl-2-(4-methoxybenzyl)-3oxopentanoate (6): clear oil; ${ }^{1} \mathbf{H}$ NMR $\left(270 \mathrm{MHz}, \mathrm{CDCl}_{3}\right) \delta_{\mathrm{H}} 0.98$ $(3 \mathrm{H}, \mathrm{t}, J=7.0 \mathrm{~Hz}), 2.20-2.60(4 \mathrm{H}, \mathrm{m}), 3.10(2 \mathrm{H}, \mathrm{d}, J=7.6 \mathrm{~Hz}), 3.76-$ $3.79(1 \mathrm{H}, \mathrm{m}), 3.77(3 \mathrm{H}, \mathrm{s}), 4.37(2 \mathrm{H}, \mathrm{t}, J=6.5 \mathrm{~Hz}), 6.80(2 \mathrm{H}, \mathrm{d}, J=$ $8.6 \mathrm{~Hz}) 7.07(2 \mathrm{H}, \mathrm{d}, J=8.6 \mathrm{~Hz}) ;{ }^{13} \mathbf{C} \mathbf{N M R}\left(67.8 \mathrm{MHz}, \mathrm{CDCl}_{3}\right): \delta_{\mathrm{C}}$ 7.4, $30.4(\mathrm{t}), 33.5,36.4,55.2,57.1,60.2,114.1,129.8,158.5,168.9$, 205.0; TLC: DCM/AcOEt $(90 / 10) \mathbf{R}_{f}=0.40$. HRMS (ESI) $(\mathrm{m} / \mathrm{z})$ : $[\mathrm{M}+\mathrm{Na}]^{+}$calcd for $\mathrm{C}_{23} \mathrm{H}_{19} \mathrm{O}_{4} \mathrm{~F}_{17} \mathrm{Na} 705.0910$, found 705.0909.

\section{Representative procedure for the Heck coupling/isomerization} reaction from intermediate 5

To a stirred solution of ester $5(0.3 \mathrm{~g}, 0.65 \mathrm{mmol})$ in dry DMF (5 mL) was added $\mathrm{NaHCO}_{3}(0.11 \mathrm{~g}, 2.0$ eq. $), \mathrm{NBu}_{4} \mathrm{Br}(0.42 \mathrm{~g}, 2.0$ eq.) and iodobenzene ( $73 \mu \mathrm{L}, 1.0 \mathrm{eq})$. The reaction mixture is then degassed with nitrogen before adding the catalyst $\left[\mathrm{Pd}(\mathrm{OAc})_{2}(14.6\right.$ $\mathrm{mg}, 10 \mathrm{~mol} \%)$ ]. After the reaction mixture was stirred 18 hours at $50{ }^{\circ} \mathrm{C}, \mathrm{H}_{2} \mathrm{O}$ was added and the resulting mixture was extracted with $\mathrm{CH}_{2} \mathrm{Cl}_{2}$. The organic layers were evaporated under reduced pressure and the residue was poured into $\mathrm{H}_{2} \mathrm{O}$ and extracted with diethyl ether. The organic layers were dried over $\mathrm{Na}_{2} \mathrm{SO}_{4}$ and evaporated under reduced pressure. The residue was purified through fluorous flash chromatography to give 7 (65\%).

1H,1H,2H,2H-Perfluorooctyl 3-oxo-5-phenylpentanoate (7): brown oil; FTIR $\left(\mathrm{cm}^{-1}\right)$ : 698 and 746 (Arom), 1144 to 1235 (CF), 1751 (ester), 1719 (ketone); ${ }^{1} \mathbf{H}$ NMR $\left(270 \mathrm{MHz}, \mathrm{CDCl}_{3}\right) \delta_{\mathrm{H}} 2.38$ $2.50(2 \mathrm{H}, \mathrm{m}), 2.83-2.91(4 \mathrm{H}, \mathrm{m}), 3.43(2 \mathrm{H}, \mathrm{s}), 4.40(2 \mathrm{H}, \mathrm{t}, J=6.4$ $\mathrm{Hz}), 7.15-7.27(5 \mathrm{H}, \mathrm{m}) ;{ }^{13} \mathbf{C ~ N M R}\left(67.8 \mathrm{MHz}, \mathrm{CDCl}_{3}\right): \delta_{\mathrm{C}} 29.4,30,3$, 44.6, 49.0, 57.1, 126.3, 128.3, 128.6, 140.3, 166.6, 201.2, ${ }^{19} \mathbf{F}$ NMR $\left(67.8 \mathrm{MHz}, \mathrm{CDCl}_{3}\right): \delta_{\mathrm{F}}-81.2(3 \mathrm{~F}),-114.1(2 \mathrm{~F}),-122.4(2 \mathrm{~F}),-123.4$ (2F), $-124.0(2 \mathrm{~F}),-126.6(2 \mathrm{~F})$.

\section{Representative procedure for the cyclization into 8-17}

To a stirred solution of $6(200 \mathrm{mg}, 0.29 \mathrm{mmol})$ in ethanol (3 $\mathrm{mL})$ was added hydrazine monohydrate $(43 \mu \mathrm{L}, 3$ eq.). The resulting mixture was stirred under reflux for 3 hours. After cooling to room temperature, the desired product $\mathbf{8}$ precipitated and was collected after filtration. The precipitate was washed with cold ethanol to give $8(66 \%)$ (in the case that products did not precipitate, they were purified through F-SPE).

3-Ethyl-4-(4-methoxybenzyl)-1H-pyrazol-5(4H)-one (8): white solid, mp: $176{ }^{\circ} \mathbf{C}$; ${ }^{1} \mathbf{H}$ NMR $\left(400 \mathrm{MHz}, \mathrm{DMSO}-d_{6}\right) \delta_{\mathrm{H}} 1.01(3 \mathrm{H}, \mathrm{t}, J$ $=7.5 \mathrm{~Hz}), 2.38(2 \mathrm{H}, \mathrm{q}, J=7.5 \mathrm{~Hz}), 3.49(2 \mathrm{H}, \mathrm{s}), 3.69(3 \mathrm{H}, \mathrm{s}), 6.80$ $(2 \mathrm{H}, \mathrm{d}, J=8.4 \mathrm{~Hz}), 7.06(2 \mathrm{H}, \mathrm{d}, J=8.4 \mathrm{~Hz}), 10.31(2 \mathrm{H}, \mathrm{bs}) ;{ }^{13} \mathbf{C}$ NMR 
$\left(100 \mathrm{MHz}, \mathrm{DMSO}-d_{6}\right): \delta_{\mathrm{C}} 13.4,17.9,26.3,54.9,99.5,113.5,128.8$, 134.0, 142.4, 157.2, 159.6; HRMS (ESI) $(\mathrm{m} / \mathrm{z})$ : $[\mathrm{M}+\mathrm{Na}]^{+}$calcd for $\mathrm{C}_{13} \mathrm{H}_{16} \mathrm{~N}_{2} \mathrm{O}_{2} \mathrm{Na} 255.1109$, found 255.1110.

3-Butyl-4-(4-methoxybenzyl)-1H-pyrazol-5(4H)-one (9): white solid, mp: $120{ }^{\circ} \mathbf{C}$; ${ }^{1} \mathbf{H}$ NMR (400 MHz, DMSO- $\left.d_{6}\right) \delta_{\mathrm{H}} 0.79(3 \mathrm{H}, \mathrm{t}, J$ $=7.3 \mathrm{~Hz}), 1.15-1.20(2 \mathrm{H}, \mathrm{m}), 1.33-1.40(2 \mathrm{H}, \mathrm{m}), 2.35(2 \mathrm{H}, \mathrm{t}, J=7.2$ $\mathrm{Hz}), 3.48$ (2H, s), $3.68(3 \mathrm{H}, \mathrm{s}), 6.79(2 \mathrm{H}, \mathrm{d}, J=8.4 \mathrm{~Hz}), 7.04(2 \mathrm{H}$, $\mathrm{d}, J=8.4 \mathrm{~Hz}), 10.31(2 \mathrm{H}, \mathrm{bs}) ;{ }^{13} \mathrm{C}$ NMR $\left(100 \mathrm{MHz}, \mathrm{DMSO}-d_{6}\right): \delta_{\mathrm{C}}$ 13.4, 17.9, 26.3, 54.9, 99.5, 113.5, 128.8, 134.0, 142.4, 157.2, 159.6; HRMS (ESI) $(\mathrm{m} / \mathrm{z}):[\mathrm{M}+\mathrm{Na}]^{+}$calcd for $\mathrm{C}_{15} \mathrm{H}_{20} \mathrm{~N}_{2} \mathrm{O}_{2} \mathrm{Na} 283.1422$, found 283.1421 .

3-Phenyl-4-(4-methoxybenzyl)-1H-pyrazol-5(4H)-one (10): white solid, mp: $212{ }^{\circ} \mathbf{C}$; ${ }^{1} \mathbf{H}$ NMR (400 MHz, DMSO- $\left.d_{6}\right) \delta_{\mathrm{H}} 3.68$ $(3 \mathrm{H}, \mathrm{s}), 3.73(2 \mathrm{H}, \mathrm{s}), 6.81(2 \mathrm{H}, \mathrm{d}, J=8.7 \mathrm{~Hz}), 7.05(2 \mathrm{H}, \mathrm{d}, J=$ $8.7 \mathrm{~Hz}), 7.30-7.45(5 \mathrm{H}, \mathrm{m}), 10.31(2 \mathrm{H}, \mathrm{bs}) ;{ }^{13} \mathbf{C}$ NMR $(100 \mathrm{MHz}$, DMSO- $\left.d_{6}\right): \delta_{\mathrm{C}} 26.6,55.0,99.9,113.7,126.4,127.7,128.7,128.8$, 130.8, 133.3, 140.0, 157.3, 160.5; HRMS (ESI) $(\mathrm{m} / \mathrm{z}):[\mathrm{M}+\mathrm{Na}]^{+}$ calcd for $\mathrm{C}_{17} \mathrm{H}_{16} \mathrm{~N}_{2} \mathrm{O}_{2} \mathrm{Na} 303.1109$, found 303.1107.

3-(2-Phenylethyl)-4-(4-methoxybenzyl)-1H-pyrazol-5(4H)-one (11): white solid, mp: $173{ }^{\circ} \mathbf{C}$; ${ }^{1} \mathbf{H}$ NMR (400 MHz, DMSO- $d_{6}$ ) $\delta_{\mathrm{H}} 2.62-2.70(4 \mathrm{H}, \mathrm{m}), 3.45(2 \mathrm{H}, \mathrm{s}), 3.68(3 \mathrm{H}, \mathrm{s}), 6.79(2 \mathrm{H}, \mathrm{d}, J=$ $8.5 \mathrm{~Hz}), 7.07(2 \mathrm{H}, \mathrm{d}, J=8.5 \mathrm{~Hz}), 7.10-7.30(5 \mathrm{H}, \mathrm{m}), 10.35(2 \mathrm{H}$, bs); ${ }^{13} \mathbf{C}$ NMR (100 MHz, DMSO- $\left.d_{6}\right): \delta_{\mathrm{C}} 26.3,26.7,55.0,100.2$, 113.5, 125.9, 128.2, 128.3, 128.9, 133.9, 140.4, 141.1, 157.2, 159.4; HRMS (ESI) $(\mathrm{m} / z)$ : $[\mathrm{M}+\mathrm{H}]^{+}$calcd for $\mathrm{C}_{19} \mathrm{H}_{21} \mathrm{~N}_{2} \mathrm{O}_{2} 309.1603$, found 309.1598 .

3-(2-Phenylethyl)-1H-pyrazol-5(4H)-one (12): white solid, mp: $197{ }^{\circ} \mathbf{C} ;{ }^{1} \mathbf{H}$ NMR $\left(400 \mathrm{MHz}, \mathrm{DMSO}-d_{6}\right) \delta_{\mathrm{H}} 2.70-2.76(2 \mathrm{H}$, m), 2.82-2.90 (2H, m), $5.23(1 \mathrm{H}, \mathrm{s}), 7.10-7.30(5 \mathrm{H}, \mathrm{m}), 11.14(2 \mathrm{H}$, bs); ${ }^{13} \mathrm{C}$ NMR (100 MHz, DMSO- $\left.d_{6}\right): \delta_{\mathrm{C}} 27.6,34.5,88.2,126.0$, 128.3, 141.2, 143.7, 160.8; HRMS (ESI) $(\mathrm{m} / \mathrm{z})$ : $[\mathrm{M}+\mathrm{Na}]^{+}$calcd for $\mathrm{C}_{11} \mathrm{H}_{12} \mathrm{~N}_{2} \mathrm{ONa} 211.0847$, found 211.0848.

1-Methyl-3-(2-phenylethyl)-1H-pyrazol-5(4H)-one (13): white solid, mp: $104{ }^{\circ} \mathbf{C}$; ${ }^{1} \mathbf{H}$ NMR (400 MHz, DMSO- $\left.d_{6}\right) \delta_{\mathrm{H}} 2.60-2.66$ $(2 \mathrm{H}, \mathrm{m}), 2.79-2.85(2 \mathrm{H}, \mathrm{m}), 3.40(3 \mathrm{H}, \mathrm{s}), 5.15(1 \mathrm{H}, \mathrm{s}), 7.10-7.30$ $(5 \mathrm{H}, \mathrm{m}), 10.73(1 \mathrm{H}, \mathrm{bs}) ;{ }^{13} \mathrm{C}$ NMR $\left(100 \mathrm{MHz}, \mathrm{DMSO}-d_{6}\right): \delta_{\mathrm{C}} 30.3$, 32.5, 34.8, 84.7, 125.7, 128.2, 128.3, 141.8, 149.2, 152.3; HRMS (ESI) $(\mathrm{m} / \mathrm{z}):[\mathrm{M}+\mathrm{H}]^{+}$calcd for $\mathrm{C}_{12} \mathrm{H}_{15} \mathrm{~N}_{2} \mathrm{O} 203.1184$, found 203.1184 .

3-(2-(4-Fluorophenyl)-ethyl)-1H-pyrazol-5(4H)-one (14): white solid, mp: $206{ }^{\circ} \mathbf{C} ;{ }^{1} \mathbf{H}$ NMR $\left(400 \mathrm{MHz}, \mathrm{DMSO}-d_{6}\right) \delta_{\mathrm{H}} 2.68-2.74(2 \mathrm{H}$, m), 2.82-2.88 (2H, m), $5.22(1 \mathrm{H}, \mathrm{s}), 7.05-7.26(4 \mathrm{H}, \mathrm{m}), 10.34(2 \mathrm{H}$, bs); ${ }^{13} \mathrm{C}$ NMR (100 MHz, DMSO- $\left.d_{6}\right): \delta_{\mathrm{C}} 27.6,33.6,88.2,115.0(\mathrm{~d}$, $\left.J_{\mathrm{C}-\mathrm{F}}=21.0 \mathrm{~Hz}\right), 130.1\left(\mathrm{~d}, J_{\mathrm{C}-\mathrm{F}}=7.8 \mathrm{~Hz}\right), 137.3\left(\mathrm{~d}, J_{\mathrm{C}-\mathrm{F}}=3.0 \mathrm{~Hz}\right)$, $143.5,160.7\left(\mathrm{~d}, J_{\mathrm{C}-\mathrm{F}}=241.1 \mathrm{~Hz}\right), 160.8 ; \operatorname{HRMS}(\mathbf{E S I})(\mathrm{m} / \mathrm{z}):[\mathrm{M}+\mathrm{Na}]^{+}$ calcd for $\mathrm{C}_{11} \mathrm{H}_{11} \mathrm{~N}_{2} \mathrm{OFNa} 229.0753$, found 229.0753.

1-Methyl-3-(2-(4-fluorophenyl)-ethyl)-4-(4-methoxybenzyl)-1Hpyrazol-5(4H)-one (15): white solid, mp: $150{ }^{\circ} \mathbf{C} ;{ }^{1} \mathbf{H} \mathbf{~ N M R}(400 \mathrm{MHz}$, DMSO- $\left.d_{6}\right) \delta_{\mathrm{H}} 2.59-2.64(2 \mathrm{H}, \mathrm{m}), 2.78-2.83(2 \mathrm{H}, \mathrm{m}), 3.39(3 \mathrm{H}, \mathrm{s})$, $5.13(1 \mathrm{H}, \mathrm{s}), 7.04-7.26(4 \mathrm{H}, \mathrm{m}), 10.85(1 \mathrm{H}, \mathrm{bs}) ;{ }^{13} \mathrm{C} \mathrm{NMR}(100 \mathrm{MHz}$, DMSO- $\left.d_{6}\right): \delta_{\mathrm{C}} 30.4,32.5,33.9,85.3,114.9\left(\mathrm{~d}, J_{\mathrm{C}-\mathrm{F}}=20.9 \mathrm{~Hz}\right), 130.1$ $\left(\mathrm{d}, J_{\mathrm{C}-\mathrm{F}}=7.8 \mathrm{~Hz}\right), 137.9\left(\mathrm{~d}, J_{\mathrm{C}-\mathrm{F}}=2.9 \mathrm{~Hz}\right), 149.1,153.3,160.7\left(\mathrm{~d}, J_{\mathrm{C}-\mathrm{F}}\right.$ $=241.0 \mathrm{~Hz}) ; \mathbf{H R M S}(\mathbf{E S I})(\mathrm{m} / \mathrm{z}):[\mathrm{M}+\mathrm{Na}]^{+}$calcd for $\mathrm{C}_{12} \mathrm{H}_{13} \mathrm{~N}_{2} \mathrm{OFNa}$ 243.0910, found 243.0908.

3-(2-(4-Methoxyphenyl)-ethyl)-1H-pyrazol-5(4H)-one (16): white solid, mp: $226{ }^{\circ} \mathbf{C}$; ${ }^{1} \mathbf{H}$ NMR (400 MHz, DMSO- $\left.d_{6}\right) \delta_{\mathrm{H}} 2.65-2.80$ (4H, m), $3.70(3 \mathrm{H}, \mathrm{s}), 5.22(1 \mathrm{H}, \mathrm{s}), 6.83(2 \mathrm{H}, \mathrm{d}, J=8.7 \mathrm{~Hz}), 7.11$ $(2 \mathrm{H}, \mathrm{d}, J=8.7 \mathrm{~Hz}), 10.83(2 \mathrm{H}, \mathrm{bs}) ;{ }^{13} \mathbf{C}$ NMR $\left(100 \mathrm{MHz}, \mathrm{DMSO}-d_{6}\right)$ : $\delta_{\mathrm{C}} 27.8,33.7,55.0,88.2,113.7,129.2,133.1,143.8,157.6,160.8$; HRMS (ESI) $(\mathrm{m} / \mathrm{z}):[\mathrm{M}+\mathrm{Na}]^{+}$calcd for $\mathrm{C}_{12} \mathrm{H}_{14} \mathrm{~N}_{2} \mathrm{O}_{2} \mathrm{Na} 241.0953$, found 241.0953.

1-Methyl-3-(2-(4-Methoxyphenyl)-ethyl)-4-(4-methoxybenzyl)1H-pyrazol-5(4H)-one (17): white solid, mp: $141{ }^{\circ} \mathbf{C} ;{ }^{1} \mathbf{H}$ NMR (400 $\left.\mathrm{MHz}, \mathrm{DMSO}-d_{6}\right) \delta_{\mathrm{H}} 2.55-2.80(4 \mathrm{H}, \mathrm{m}), 3.39(3 \mathrm{H}, \mathrm{s}), 3.71(3 \mathrm{H}, \mathrm{s})$, $5.12(1 \mathrm{H}, \mathrm{s}), 6.83(2 \mathrm{H}, \mathrm{d}, J=8.7 \mathrm{~Hz}), 7.12(2 \mathrm{H}, \mathrm{d}, J=8.7 \mathrm{~Hz}), 10.68$ $(1 \mathrm{H}, \mathrm{bs}) ;{ }^{13} \mathrm{C}$ NMR $\left(100 \mathrm{MHz}, \mathrm{DMSO}-d_{6}\right): \delta_{\mathrm{C}} 30.6,32.5,33.9,54.9$, 84.7, 113.6, 129.2 133.7, 149.2, 157.4; HRMS (ESI) $(\mathrm{m} / \mathrm{z})$ : $[\mathrm{M}+\mathrm{Na}]^{+}$ calcd for $\mathrm{C}_{13} \mathrm{H}_{16} \mathrm{~N}_{2} \mathrm{O}_{2} \mathrm{Na} 255.1109$, found 255.1107.

\section{RESULTS AND DISCUSSION}

We initially focused on the synthesis of fluorous substrates 1-5 for the Heck reaction. The starting Baylis-Hillman adducts 1-4 were synthesized according to our previous protocol. ${ }^{23}$ Commercially available fluorous acrylate was coupled to an aliphatic or aromatic aldehyde using DABCO in neat conditions at room temperature for $48 \mathrm{~h}$ (disappearance of the starting acrylate was monitored by TLC) to produce the corresponding fluorous-tagged derivatives 1-4 (Scheme 2).

Purification was accomplished using a fluorous silica gel chromatography. The crude reaction mixture was added to the fluorous silica column and the excess reagents and other non fluorous products were eluted following a fluorophobic wash (methanol and water in 80:20 ratio) whereas the fluorous adduct was retained onto the SPE cartridge until elution with a fluorophilic solvent $\left(100 \% \mathrm{CH}_{2} \mathrm{Cl}_{2}\right)$. This purification method was rapid (10 min per compound) and generated desired compounds $1-4$ with a $72-85 \%$ yield. In some instances, ${ }^{1} \mathrm{H}$ NMR analyses also revealed the presence of a perfluorinated alcohol, resulting in a hydrolysis of the $\mathrm{BH}$ adducts into a dioxanone derivative due to the excess of aldehyde. However, these

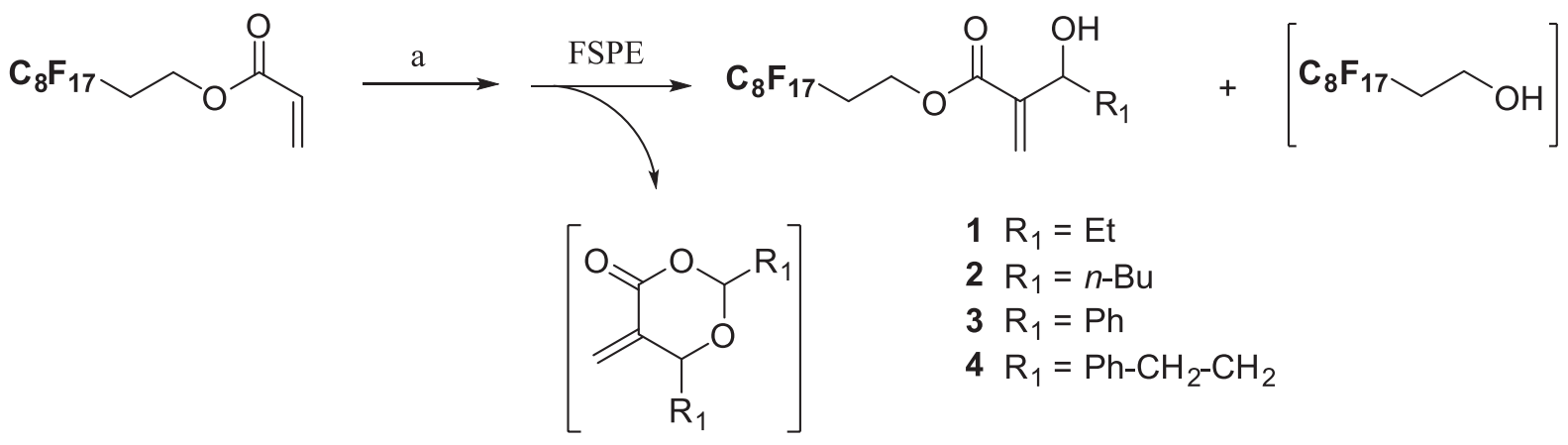




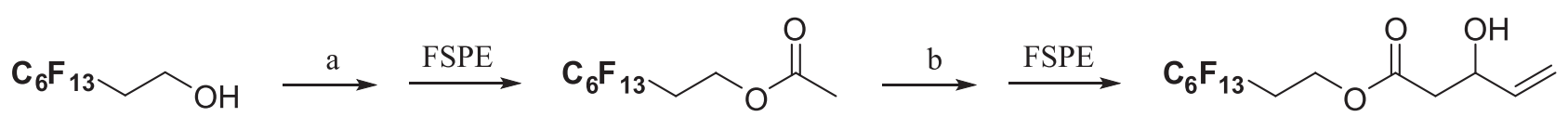

Scheme 3. (a) AcCl (1.2 eq.), TEA, DCM, r.t., 18 h (98\%); (b) i. LHMDS (1.1 eq.), THF, $-78^{\circ} \mathrm{C}, 45$ min.; ii. Acrolein (1.5 eq.), $\mathrm{THF},-78^{\circ} \mathrm{C}, 10 \mathrm{~min} . ; \mathrm{iii}$. TMSCl (1.0 eq.), THF, $-78^{\circ} \mathrm{C}, 4$ h. $(70 \%)$

two byproducts did not induce further purification steps since the dioxanone was removed by the fluorophobic wash, and the fluorous alcohol did not interfere in the following steps.

Synthesis of perfluorinated hydroxypentenoate 5 was accomplished conveniently in two steps as outlined in scheme 3 .

Polyfluorooctanol was used as a perfluorous tag and gave under standard conditions $\mathrm{s}^{30}$ (acetyl chloride and triethylamine in dichloromethane) the corresponding acetate in a very good yield (98\%) after F-SPE purification. We next investigated the aldol condensation of this acetate with acrolein. Formation of lithium enolate was attempted with LDA or LHMDS as a base in THF at -78 or $-50{ }^{\circ} \mathrm{C}$. During these first attempts ester hydrolysis occurred and was easily confirmed by the presence of perfluorous alcool by ${ }^{1} \mathrm{H}$ NMR analysis. The best result, $70 \%$ purified yield of 5 was obtained by treatment of perfluorinated acetate with LHMDS (1.1 eq.), followed by the addition of acrolein then trimethylsilyl chloride (1.0 eq.). Actually, it was shown that the yield could be significantly improved by the addition of trimethylsilyl chloride to stabilize the intermediate aldolate. ${ }^{30}$

With these perfluorinated allylic alcohols 1-5 in hand, we next focused on the tandem Mizoroki-Heck cross-coupling reaction and double-bond isomerization. This tandem Heck-isomerization reaction is well documented with allylic alcohols ${ }^{31,32}$ and Baylis-Hillman adducts. ${ }^{33-36}$ During this reaction, Heck reaction with an adequate palladium catalyst leads to the formation of the $\beta$-keto ester required for the end of the sequence.

Basavaiah and Muthukumaran ${ }^{34}$ described a very efficient Heck reaction (using the 'Jeffery protocol') ${ }^{37}$ to convert Baylis-Hillman adducts into $\alpha$-benzyl- $\beta$-keto esters avoiding the formation of mixture of products. ${ }^{34}$ Based on these conditions and after a few optimization, the fluorous Baylis-Hillman adduct 1 led, in the presence of 4-iodoanisole (3.0 eq.), $\mathrm{NaHCO}_{3}$ (3.5 eq.), $\mathrm{Bu}_{4} \mathrm{NBr}$ (1.0 eq.) and $\mathrm{Pd}(\mathrm{OAc})_{2}(5$ $\mathrm{mol} \%$ ), in refluxing THF to the predominant formation of the $\beta$-keto ester 6 with a good yield (90\%) after F-SPE purification (Scheme 4). However, ${ }^{1} \mathrm{H}$ NMR analysis of the crude material revealed the presence of small amounts of byproduct A (about 10\%).
Similarly, fluorous allylic alcohol 5 was submitted to the same reaction conditions in order to introduce a second diversity. The reaction conditions were optimized using 5 and iodobenzene as the coupling partner. The best results were obtained when hydroxypentenoate 5 reacted with 4-fluoro-iodobenzene (1.0 eq.) in DMF at $50{ }^{\circ} \mathrm{C}$ for 18 hours in the presence of $\mathrm{Pd}(\mathrm{OAc})_{2}(10 \mathrm{~mol} \%), \mathrm{NaHCO}_{3}$ as base and $\mathrm{Bu}_{4} \mathrm{NBr}$ (1.0 eq.), thus affording the required derivative 6 in satisfactory isolated yield $(65 \%)$. After F-SPE purification, presence of small amounts of compound B and prefluorinated alcohol -due to ester hydrolysis- (about $10 \%$ in each case) was observed by ${ }^{1} \mathrm{H}$ NMR analysis of the crude material. However, these byproducts did not induce further purification step since they did not interfere in the following step.

Once the starting materials were prepared and the Heck coupling and isomerization reactions to obtain $\beta$-keto esters validated, we planned to obtain a library of pyrazolones (Tables 1 and 2). The intermediates $\beta$-keto esters obtained from Baylis-Hillman adducts were condensed with hydrazine. The reaction was carried out in EtOH under reflux for 3 hours. After cooling to room temperature, the cyclized products $8-11$ precipitated out from the reaction medium since they had low solubility in such a solvent, and were collected in a moderate overall yield by filtration.

Compounds 12-17 were obtained in a similar manner starting from intermediate 5 . The cyclized products did not precipitated out from the reaction medium when using methylhydrazine (compounds 13, 15 and 17). In this case, the reaction media was evaporated to remove solvent and the crude mixture was taken up with dichloromethane and washed with water. The residue was then purified through F-SPE to afford the substituted pyrazolones in the fluorophobic wash and complete removal of fluorous products in the fluorophilic wash. The efficiency of this purification step was good regarding the ${ }^{1} \mathrm{H}$ NMR spectra of the final compounds.

All the products were identified by ${ }^{1} \mathrm{H},{ }^{13} \mathrm{C}$ NMR and mass spectrometry. The $1 \mathrm{H}$-pyrazolin-5-one presents three tautomeric forms due to their keto-enolic tautomerism, the full aromatic enol form $(\mathrm{OH}$ tautomer) being the predominent one (Figure 2).<smiles>C=C(C(=O)OCCC(F)F)C(O)CC</smiles>
1<smiles>C=CC(O)CC(=O)OCC[SH](C)(F)(F)F</smiles>
5<smiles>CCC(=O)C(Cc1ccc(OC)cc1)C(=O)OCCC(F)C(F)(F)F</smiles><smiles>CCC(=O)C(C)C(=O)OCCC(F)F</smiles>

7<smiles>O=C(CCc1ccccc1)CC(=O)OCCC(F)(F)F</smiles><smiles>O=C(CC(O)C=Cc1ccccc1)OCCC(F)(F)F</smiles>

Scheme 4. (a) 4-Iodoanisole (3.0 eq.), $\mathrm{NaHCO}_{3}\left(3.5\right.$ eq.), $\mathrm{Bu} \mathrm{H}_{4} \mathrm{NBr}\left(1.0 \mathrm{eq}\right.$.), $\mathrm{Pd}(\mathrm{OAc})_{2}(5 \mathrm{~mol} \%), \mathrm{THF}$, reflux, 18 h (90\%); (b) Iodobenzene (1.0 eq.), $\mathrm{NaHCO}{ }_{3}$ (2.0 eq.), $\mathrm{Bu} u_{4} \mathrm{NBr}\left(2.0 \mathrm{eq}\right.$.), $\mathrm{Pd}(\mathrm{OAc})_{2}(10 \mathrm{~mol} \%), \mathrm{DMF}, 50^{\circ} \mathrm{C}, 18 \mathrm{~h}(65 \%)$ 
Table 1. Synthesis of 3,4-disubstituted pyrazolones

1. Iodoanisole
$\mathrm{Pd}(\mathrm{OAc})_{2}(5 \mathrm{~mol} \%)$
$\mathrm{NaHCO}, \mathrm{Bu}_{4} \mathrm{NBr}$
$\mathrm{THF}, \mathrm{Reflux}$

${ }^{\mathrm{a}}$ Calculated from allylic alcohols 1-4.

Table 2. Synthesis of 1,3-disubstituted pyrazolones

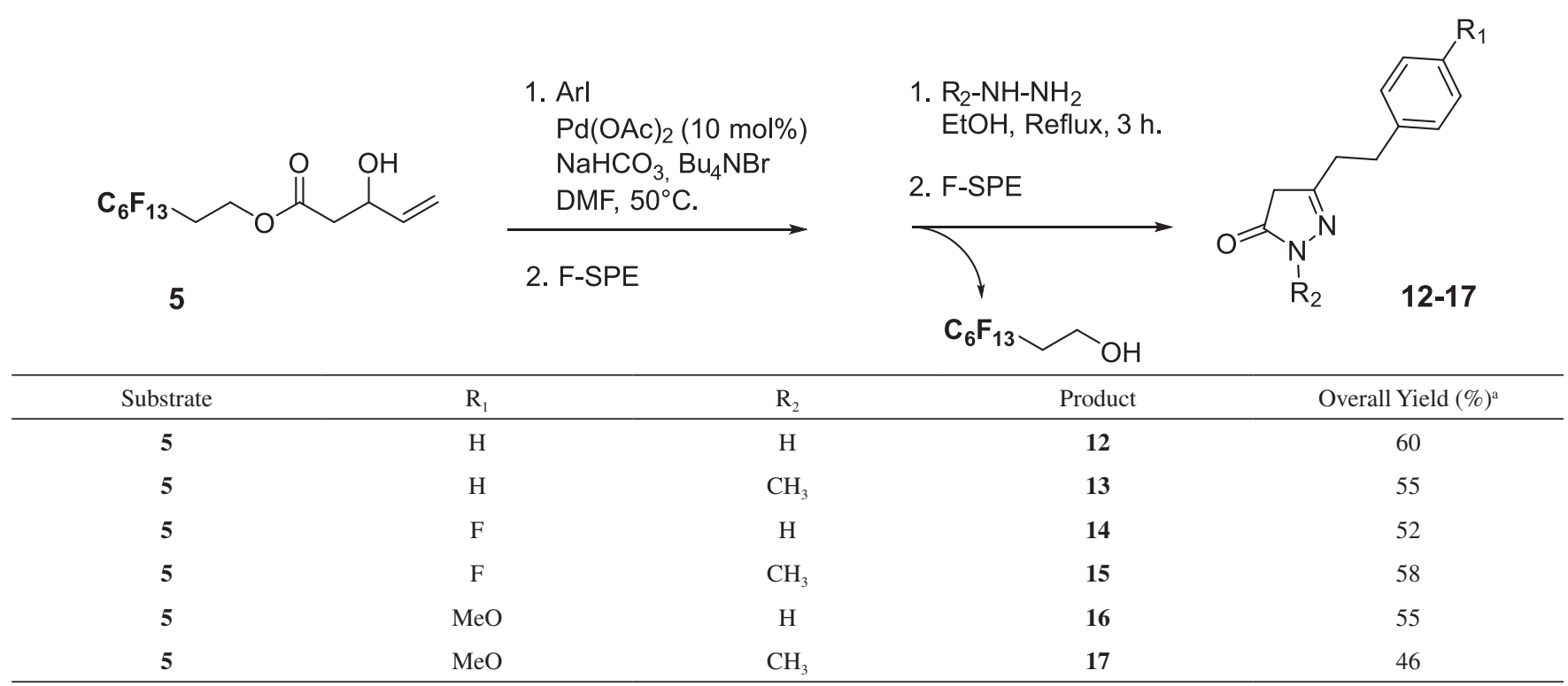

${ }^{\text {a }}$ Calculated from starting allylic alcohol $\mathbf{5}$.

Analysis of these compounds by NMR was not so easy in $\mathrm{CDCl}_{3}$ due to their existence as a mixture of three tautomeric forms ( $\mathrm{CO}$ tautomer being predominent), whereas in DMSO- $d_{6}$ they appeared as a single $\mathrm{OH}$ tautomer form. The $\mathrm{OH}$ tautomeric form was confirmed by ${ }^{1} \mathrm{H}$ and ${ }^{13} \mathrm{C}$ NMR spectra analyses. Actually, for 12 as an example, a two protons broad signal between 9 and $11 \mathrm{ppm}$ due to the presence of two exchangeable protons $(\mathrm{OH}$ and $\mathrm{NH})$, and a $\mathrm{H}-4$ signal at $5.23 \mathrm{ppm}$ confirmed this tautomeric form. Furthermore, the ${ }^{13} \mathrm{C}$ NMR spectrum of this compound is also in accordance with the $\mathrm{OH}$ tautomer by the presence of a $\mathrm{C}-4$ signal at $88.2 \mathrm{ppm}$. These chemical shifts are in accordance with the literature..$^{38,39}$

\section{CONCLUSIONS}

In summary, ten substituted pyrazolone derivatives were easily synthesized from perfluorinated allylic alcohols, via a straightforward<smiles>[R]C1=NN([R])C(=O)C1[R2]</smiles>

CO tautomer<smiles>[R]c1nn([R])c(O)c1[R]</smiles>

$\mathrm{OH}$ tautomer<smiles></smiles>

NH tautomer<smiles>Oc1cc(CCc2ccccc2)n[nH]1</smiles>

12

Figure 2. Tautomerism of the pyrazolin-5-one derivatives 
two steps reaction involving a Heck-coupling/isomerization reaction and subsequent condensation of hydrazines that allows for a cyclorelease of the final products with a good purity. The combination of this approach and F-SPE purification represents an attractive method that has potential for automation to prepare libraries of compounds for high-throughput screening.

\section{SUPPLEMENTARY MATERIAL}

${ }^{1} \mathrm{H}$ and ${ }^{13} \mathrm{C}$ NMR spectra for compounds $8-17$ are available at http://quimicanova.sbq.org.br, in pdf file with free access.

\section{ACKNOWLEDGEMENTS}

The authors thank the Brazilian program Ciência Sem Fronteiras and $\mathrm{CNPq}$ for financial support and fellowships. We gratefully acknowledge the CRMPO for mass spectrometry experiments.

\section{REFERENCES}

1. Elguero, J. In Comprehensive Heterocyclic ChemistryII; Katritzky, A. R., Rees, C. W., Scriven, E. F. V., eds.; Pergamon Press: Oxford, 1996; Vol. 3, p 1 .

2. Yet, L. In Comprehensive Heterocyclic ChemistryII; Katritzky, A. R., Ramsden, C. A., Scriven, E. F. V., Taylor, R. J. K., eds.; Elsevier: Oxford, 2008; Vol. 4, p 1.

3. Fustero, S.; Sanchez-Rosello, M.; Barrio, P.; Simon-Fuentes, A.; Chem. Rev. 2011, 111, 6984.

4. Keri, R. S.; Chand, K.; Ramakrishnappa, T.; Nagaraja, B. M.; Arch. Pharm. Chem. Life Sci. 2015, 348, 299.

5. Brogden, R. N.; Drug 1986, 32, 60.

6. Kuo, S.-C.; Huang, L.-J.; Nakamura, H.; J. Med. Chem. 1984, 27, 539.

7. Volz, M.; Kellner, H. M.; J. Clin. Pharmacol. 1980, 10, 299 S.

8. Bondock, S.; Rabie, R.; Etman, H. A.; Fadda, A. A.; Eur. J. Med. Chem. 2008, 43, 2122.

9. Orrling, K. M.; Jansen, C.; Vu, X. L.; Balmer, V.; Bregy, P.; Shanmugham, A.; England, P.; Bailey, D.; Cos, P.; Maes, L.; Adams, E.; van den Bogart, E.; Chatelain, E.; Ioset, J.-R.; van de Stolpe, A.; Zorg, S.; Veerman, J.; Seebeck, T.; Sterk, G. J.; de Esch, I. J. P.; Leurs, R.; J. Med. Chem. 2012, 55, 8745.

10. Sujatha, K.; Shanthi, G.; Selvam, N. P.; Manoharan, S.; Perumal, P. T.; Rajendran, M.; Bioorg. Med. Chem. Lett. 2009, 19, 4501.

11. Mahajan, S. S.; Scian, M.; Sripathy, S.; Posakony, J.; Lao, U.; Loe, T. K.; Leko, V.; Thalhofer, A.; Schuler, A. D.; Bedalov, A.; Simon, J. A.; J. Med. Chem. 2014, 57, 3283.

12. Okuyama, S.; Morita, M.; Sawamoto, A.; Terugo, T.; Nakajima, M.; Furukawa, Y.; Pharmaceuticals 2015, 8, 176.
13. Jiao, S.-S.; Yao, X.-Q.; Liu, Y.-H.; Wang, Q.-H.; Zeng, F.; Lu, J.-J.; Liu, J.; Zhu, C.; Shen, L.-L.; Liu, C.-H.; Wang, Y.-R.; Zeng, G.-H.; Parikh, A.; Chen, J.; Liang, C.-R.; Xiang, Y.; Bu, X.-L.; Deng, J.; Li, J.; Xu, J.; Zeng, Y.-Q.; Xu, X.; Xu, H.-W.; Zhong, J.-H.; Zhou, H.-D.; Zhou, X.-F.; Wang, Y.-J. Proc. Natl. Acad. Sci. U. S. A. 2015, 112, 5225.

14. Hadi, V.; Koh, Y.-H.; Sanchez, T. W.; Barrios, D.; Neamati, N.; Jung, K. W.; Bioorg. Med. Chem. Lett. 2010, 20, 6854.

15. Laleu, B.; Gaggini, F.; Orchard, M.; Fioraso-Cartier, L.; Cagnon, L.; Houngninou-Molango, S.; Gradia, A.; Duboux, G.; Merlot, C.; Heitz, F.; Szyndralewiez, C.; Page, P.; J. Med. Chem. 2010, 53, 7715.

16. Knorr, L.; Chem. Ber. 1884, 17, 546.

17. Claisen, L.; Lowman, O.; Chem. Ber. 1887, 20, 651.

18. Meldrum, A. N.; J. Chem. Soc., Trans. 1908, 93, 598.

19. Oikawa, Y.; Sugano, K.; Yonemitsu, O.; J. Org. Chem. 1978, 43, 2087.

20. Basavaiah, D.; Muthukumaran, K.; Tetrahedron 1998, 54, 4943.

21. Amaral, P. A.; Petrignet, J.; Gouault, N.; Agustini, T.; Lohézic-Le Dévéhat, F.; Cariou, A.; Grée, R.; Eifler-Lima, V. L.; David, M.; J. Braz. Chem. Soc. 2009, 20, 1687.

22. Nicolaou, K. C.; Hanko, R.; Hartwig, W.; Handbook of combinatorial chemistry. Drugs, catalysts, materials; Wiley-VCH: Weinheim, 2002; Vol. 1, p. 1114.

23. Le Lamer, A.-C.; Gouault, N.; David, M.; Boustie, J.; Uriac, P.; J. Comb. Chem. 2006, 8, 643.

24. Horhant, D.; Le Lamer, A.-C.; Boustie, J.; Uriac, P.; Gouault, N.; Tetrahedron Lett. 2007, 48, 6031.

25. Le Lamer, A.-C.; Authier, H.; Rouaud, I.; Coste, A.; Boustie, J.; Pipy, B.; Gouault, N.; Bioorg. Med. Chem. Lett. 2014, 24, 3819.

26. Studer, A.; Hadida, S.; Ferrito, R.; Kim, S.-Y.; Jeger, P.; Wipf, P.; Curran, D. P.; Science 1997, 275, 823.

27. Zhang, W.; Chem. Rev. 2004, 104, 2531.

28. Curran, D. P.; Luo, Z.; J. Am. Chem. Soc. 1999, 121, 9069

29. Eifler-Lima, V. L.; Amaral, P. A.; Rev. Virtual Quim. 2010, $2,298$.

30. Eames J.; Khanom H.; Molecules 2004, 9, 266.

31. Larock, R. C.; Leung, W. Y.; Stolz-Dunn, S.;Tetrahedron Lett.1989, 30, 6629.

32. Bollikonda, S.; Mohanarangam, S.; Jinna, R. R.; Kandirelli, V. K. K.; Makthala, L.; Sen, S.; Chaplin, D. A.; Lloyd, R. C.; Mahoney, T.; Dahanukar, V. H.; Oruganti, S.; Fox, M. E.; J. Org. Chem. 2015, 80, 3891.

33. Ferrera, B. R. V.; Pirovani, R. V.; Souza-Filhi, L. G.; Coelho, F.; Tetrahedron 2009, 65, 7712 .

34. Basavaiah, D.; Muthukumaran, K.; Tetrahedron 1998, 54, 4943.

35. Kulkarni, B. A.; Ganesan, A.; J. Comb. Chem. 1999, 1, 373.

36. Coelho, F.; Veronese, D.; Pavam, C. H.; De Paula, V. I.; Buffon, R.; Tetrahedron 2006, 62, 4563.

37. Jeffery, T. J.; J. Chem. Soc., Chem. Commun. 1984, 1287.

38. Isaad, J.; Tetrahedron 2013, 69, 2239.

39. Metwally, M. A.; Bondock, S. A.; El-Desouky, S. I.; Abdou, M. M.; Int. J. Modern Org. Chem. 2012, 1, 19. 